\title{
A Dynamic Multiple Cluster-Head Clustering Algorithm for Wireless Networks
}

\author{
Lu Fei \\ Shenyang Normal University \\ Software College, Shenyang Normal University \\ Shenyang, China \\ lu859290995@163.com \\ * Corresponding Author \\ Zhao Yongyi \\ Shenyang Normal University \\ Software College, Shenyang Normal University \\ Shenyang, China \\ 283631215@qq.com
}

\author{
Liu Yi \\ Shenyang Normal University \\ Software College, Shenyang Normal University \\ Shenyang, China \\ 283631215@qq.com
}

\begin{abstract}
At present, the technology of wireless sensors develops rapidly, there are more and more studies in this area, especially the researches of sensor node energy. In this paper, we propose a new algorithm which named a dynamic multiple cluster-head clustering algorithm. In this algorithm, according to the energy of each node and nodes density, we divide the networks into seveal cluster structure. In each cluster structure, the cluster structure is divided into several layers according to the sink node hops. Each layer is independent from each other, then in the layer, we select a cluster head node, and only the cluster-head can communicates with each other. The rest of nodes will be selected as the cluster head nodes, the remaining nodes of each layer are regarded as children nodes cluster head nodes of cluster head nodes. In this way, we can get a new network topology structure and reselect the cluster head irregularly according the method which mentioned above. The algorithm that we proposed can guarantee balanced energy consumption, and prolong the lifetime of the network.
\end{abstract}

Keywords- Wireless Sensor Networks; Dynamic; Multiple Cluster-Head; Clustering Algorithm; Energy Balance;

\section{INTRODUCTION}

With the development of society and the progress of science, wireless sensor networks has been developed rapidly, and has become a hot research topic at home and abroad. Wireless sensor networks is a distributed sensing networks, which is composed of a large number of static or mobile sensor nodes in the form of self organization and multi hop wireless network, which is used to sense, data collecting, data processing and transmit the information on the perceived object within the geographic area covered by the network ${ }^{[1]}$, and eventually put these information and send them to the owners of the networks. Wireless sensor networks can be widely used in military reconnaissance, environment monitoring, medical care, agricultural breeding and other fields. [2] With the continuous development of wireless sensor networks, wireless sensor network has become a hot. From the point of network topology, WSN can be divided into two categories: planar structure and clustering structure. In the plane structure, the position of WSN nodes is equal. In the clustering structure, however, nodes in the network are divided into several clusters, and each cluster is composed of a cluster head node and a plurality of member nodes. Cluster head is responsible for the management and control of the cluster nodes, and is responsible for the data collection and data forwarding among clusters. Compared with the planar structure, WSN has the advantages of high energy efficiency, good scalability, and so on. But how to select the cluster head, classify cluster, need a suitable clustering algorithm to solve it. The typical clustering algorithms are HEED, LEACH, and PEGASIS, etc. The following is the introduction of the HEED algorithm.

HEED ${ }^{[3-5]}$ is a clustering protocol which has a fixed cluster radius. As a measure of cost in clustering communication, Heed chooses the cluster-head depending on two parameters. The main parameter influcenced by the remaining energy, the node which has more remaining energy will be selected as a temporary cluster-head, and the algorithm's convergence rate will be higher. Coparameter concludes node proximity and node density. Multiple cluster head nodes in the same cluster compete for the final cluster head through the average energy level of the AMRP. For member nodes in the range of multiple clusters, the final cluster is selected according to the secondary parameter AMRP. HEED takes into account the survival time, scalability and load balance, so that the distribution of nodes is more uniform. However, the energy consumption of the nodes which near the sink node is still not resolved, and there will be a lot of overhead when detect the energy consumption or exchange energy consumption information.

In order to solve the problem mentioned above, we propose the dynamic multiplecluster-head clustering algorithm. Firstly, sink nodes choose the cluster-head according to the energy of each node and nodes density, and divide the networks into seveal cluster structure ${ }^{[6]}$. The nodes which have more energy and more intensive are the cluster head node. For the member nodes in the range of multiple clusters, the final cluster is selected according to the count of energy. In each cluster structure, we divide 
the cluster structure into several layers ; according to each node of energy situation, select a cluster-head node in each layer, the remaining nodes which have more energy as a priority is the cluster-head node, the remaining nodes of each layer are regarded as cluster-head nodes of children nodes, in this way, we can get a new network topology structure. $^{[7-9]}$ And reselect the cluster-head irregularly according to energy situation of cluster-head, and reform a new network topology structure, guaranteeing a balanced energy consumption, so as to prolong the lifetime of the network.

\section{THE ALGORITHM}

\section{A. Algorithm Description}

We have to make some definitions before the introduction of the algorithm.

Definition 1: Sink node: Also called sink node, is mainly responsible for the sensor network and external network connections, can be seen as the gateway node.

Definition 2: Ordinary nodes: All nodes, except the sink node, in the whole wireless sensor network are called ordinary nodes.

Definition 3: Cluster head node: in a cluster, selects a node as a cluster head firstly, all nodes in the cluster pass the data to the cluster head, and the cluster head forwards the data to the next hop or directly to the sink node.

Definition 4: The cluster node: in one cluster, the nodes are called the cluster nodes except cluster-haed node, and the nodes of the cluster are the child nodes of the cluster head.

We will describe the algorithm in this part:

\section{1) Clustering}

In a network consisting of sink nodes and several nodes, the first sink node broadcasts messages to all nodes in the network. All nodes in the command network transmit messages to the sink node, the message format is (ID, Hop, E, Nodes), where ID is the number of nodes, Hop is the number of hops to the sink node, and $\mathrm{E}$ is the remaining energy of the node, Nodes is the number of adjacent nodes. The sink nodes choose the cluster-head according to the energy of each node and nodes density, and divide the networks into seveal cluster structure. The nodes which have more energy and more intensive are the cluster head node. For the member nodes in the range of multiple clusters, the final cluster is selected according to the count of energy.

\section{2) Clustering in Cluster}

In any cluster, the sink node hops for all nodes under each node layers, Hop-1 node for the first layer, Hop-2 nodes for the second layer, and so on, the entire network is divided into several layers, and here we assume that all nodes which are in the same layer can communicate with each other. Then the sink node selects the cluster-head node in each layer, and the sink node selects the node with high energy as the cluster-head node, and the other nodes are connected with the cluster-head node in the layer. The remaining nodes are the children of the cluster-head node, which can only be connected to the cluster-head node in the layer, and can not connect to other nodes. Take the first layer as an example, all nodes in the first layer are all nodes, and the energy value of all ordinary nodes is compared. The highest energy value is selected as the cluster-head node. After the cluster-head node is selected, the node sends a message to all nodes in the first layer, so that the remaining nodes in the first layer do the child node of the cluster-head, and named as the cluster node. In the same way, the rest layer is divided into cluster processing and the cluster-head node of each layer can communicate, so as to form a concise and simple network topology structure.

\section{3) Change the Cluster-Head}

As the cluster-head node is to fuse the information of its child nodes, the energy consumption of the cluster-head is larger. So we set a threshold for residual energy value, when the residual energy of a cluster-head node value is less than the threshold, send a request to the sink node, the sink node received the message after processing, re networking. If a cluster-head be low- energy, sink node will re-clustering; when a cluster-head is low-energy, according to the size of the residual energy re selected a new cluster-head nodes. Other nodes, as the child nodes of the new cluster-head nodes, form a new network topology, which can make all the nodes energy balance and prolong the network lifetime.

\section{B. Algorithms for Example}

In order to better understand the algorithm, the operation process of the algorithm is given, and the topology structure of wireless sensor network is shown in "Fig. 1", where the node is sink node and the other nodes are ordinary nodes.

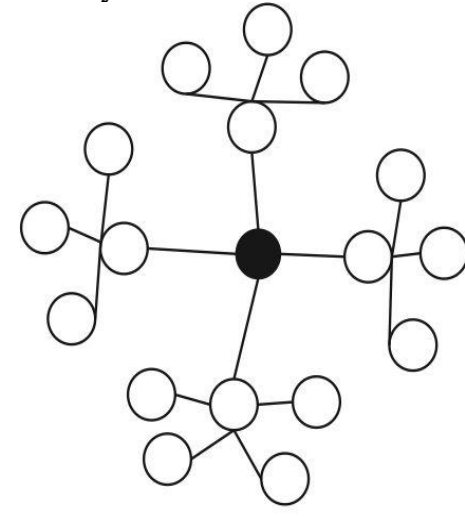

sink node
common node

Figure 1. Network Topology

\section{1) Clustering}

The algorithm starts with the sink node to broadcast messages to the entire network, and requires each node feedback node information, the information format is (ID, Hop, E, Nodes), where ID is the number of nodes, Hop is the number of hops to the sink node, $\mathrm{E}$ is the residual energy. Nodes is the number of adjacent nodes.After the sink node receives all the information from all nodes, Then sink nodes choose the cluster-head according to the energy of each node and nodes density, and divide the networks into seveal cluster structure. The nodes, which have more energy and more intensive, are the cluster head node. For the member nodes in the range of multiple clusters, the final cluster is selected according to the count of energy. Afterwards, the clustering diagram is as shown in "Fig. 2". 


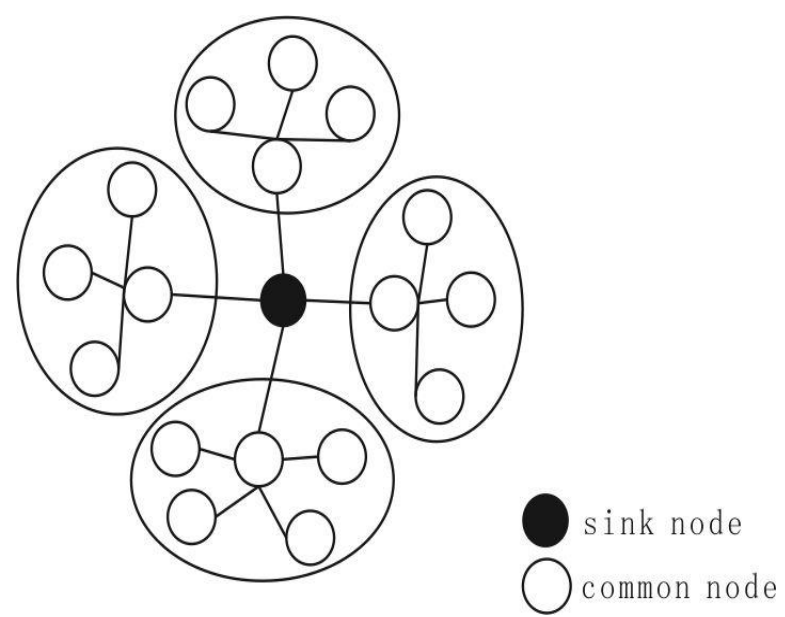

Figure 2. Clustering Diagram

\section{2) Cluster-Head Selection}

In any cluster, the cluster structure is divided into several layers according to the sink node hops, the sink node is layered, Hop-1 node is the first layer, and Hop-2 node is the second layer... After the hierarchy as shown in "Fig 3." 2,3,4 nodes are for the first layer, 5,6,7, nodes are for the second layer, 8,9 nodes is for the third layer.In any cluster, the sink nodes are chosen, and the remaining energy values of each layer are compared with the sink node, and the node with high energy is chosen as the cluster-head, according to the network topology of Figure 1 , we can know that the remaining energy of the first layer of 3 nodes is the highest. So the 3 node is the first layer of the cluster-head node, the second layer head node is node 6 , and the third layer cluster-head node is node $8 .{ }^{[10]}$
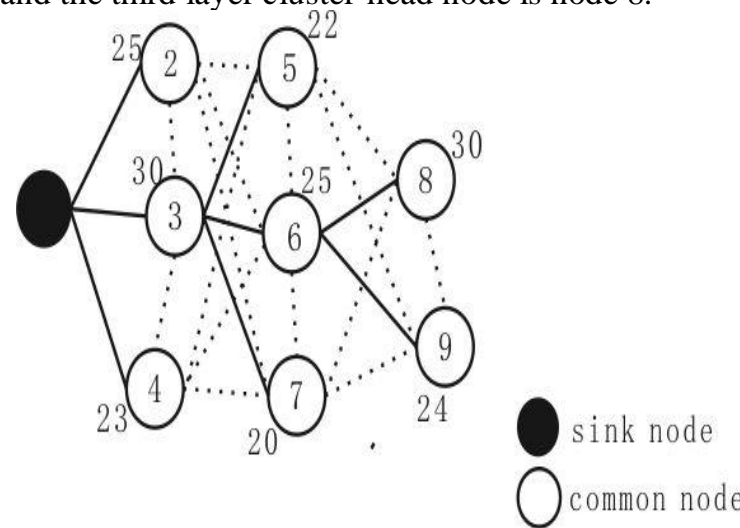

\section{- the connected} -..communication

Figure 3. Network Topology

\section{3) Networking}

After the selection of the cluster-head node, the sink node sends a message to each layer of the cluster-head node, the node of the cluster-head of each layer is organized, and the head node of the cluster is connected with each layer. This is formed by the cluster-head node to form the main road, and then each layer of the head node to the other nodes in the layer of the network message, each layer of the cluster-head node outside the other nodes to the cluster-head node request to join the network, The cluster-head node detects a message and finds that it is a node in its own layer, and agrees to join the network, otherwise it is rejected. In the first layer, the 3 head node sends a message to the network, and the $1,2,4,5,6,7$ nodes receives the message, because the 1 node is not processed by the sink node, and the 2,4 nodes is the first layer of the cluster node, which is connected with the cluster-head node; the node of 5,7 is not to do the processing of the second layer cluster, and the second node is the 3 layer cluster-head node, which is connected with the head node of cluster-head of 6.In the second layer, the head node of the cluster-head is sent to the network message, the 2,3,4,5,7,8,9 nodes receives the message, and the 2,3,4 nodes is the node in the first layer, so it is not processed;The 5,7 nodes is a cluster of second nodes, which is connected with node 6 , and the 8 is connected with the third layer head node, which is connected with 6 nodes, and the 9 is connected with the 8 . In this way we obtain a multicluster-head of networks topology. replace the cluster-head. ${ }^{[1]]}$ To form a new network topology as shown in "Fig 4.".
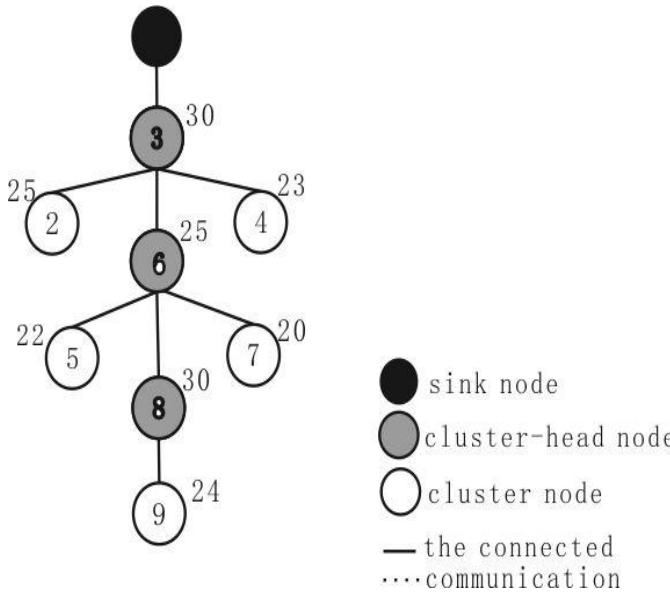

Figure 4. the New Network Topology

\section{4) Replace Cluster-Head}

Because the cluster-head node in the work process to receive the data fusion processing ${ }^{[12]}$, the energy will be very fast, so we should be appropriate to replace the head node. We first set a threshold, when the cluster-head node residual energy value is below this threshold, the clusterhead node to the sink node sends just for cluster-head nodes request. After receiving the information from the sink node, the message is broadcast to the entire network, and the sink node is selected as a new cluster-head node, and the new cluster-head node is connected with each other. In each layer, the remaining nodes apply to join the network to form a new topological structure. Assume that the energy threshold is the residual energy of node number 10,6 values less than 10 , requesting the sink node to the network. To form a new network topology is as shown in "Fig. 5". 


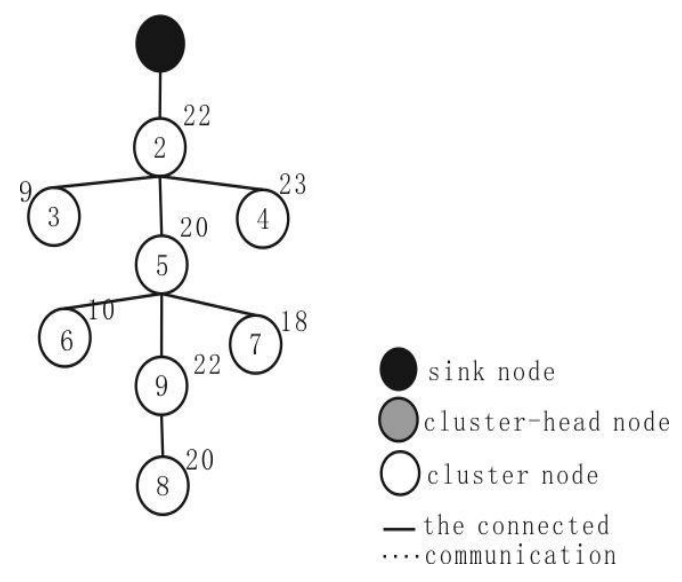

Figure 5. the New Network Topology

\section{CONCLUSION}

This paper introduces a dynamic cluster-head algorithm for wireless senor networks, the algorithm divide the networks into seveal cluster structure according to the energy of each node and nodes density. In each cluster structure, according to each node of energy situation, select a cluster-head node, in each cluster structure, the cluster structure is divided into several layers according to the sink node hops the remaining nodes which have more energy as a priority is the clusterhead node, the remaining nodes of each layer are regarded as cluster-head nodes of children nodes, in this way, we can get a new network topology structure and reselect the cluster-head irregularly according to energy situation of cluster-head, and reform a new network topology structure, guaranteeing a balanced energy consumption, so as to prolong the lifetime of the network.

Wireless sensor network is a network of limited energy, if the energy of some of the nodes in the network may consume too quickly, and lead to some of the nodes fail, and even affect the operation of the entire network.Therefore, we must consider how to solve the problem of energy consumption of nodes, and node energy consumption can be balanced, thus extending the lifetime of the network. The algorithm solves this problem. HEED takes into account the survival time, scalability and load balance, so that the distribution of nodes is more uniform. However, the energy consumption of the nodes which near the sink node is still not resolved, and there will be a lot of overhead when detect the energy consumption or exchange energy consumption information.Comparing with HEED, the algorithm that we proposed can distribute the energy consumption to the whole network better So that the energy of each cluster in the whole wireless network can be as much as possible with the same proportion of consumption. At the same time, the ynamic multiplecluster-head clustering algorithm can solve the problem that the nodes energy consumption too fast, and prolong the lifetime of the networks more efficient.

\section{REFERENCE}

[1] M Shakir.Wireless sensor networks [M].France: LAP Lambert Academic Publishing, 2011.

[2] R Faludi.Building wireless sensor networks: with zigbee, xbee, arduino, and processing M]. USA: O'Reilly Media, 2011.

[3] R Pawlak B Wojciechowski, M Nikodem. New simplified heed algorithm for wireless sensor networks [D]. 17th Conference, CN 2010, Ustroń, Poland, June 15-19, 2010. Proceedings.

[4] Guangbing Xiao, Ning Sun, Liya Lv et al. A heed-based study of cell-clustered algorithm in wireless sensor network for energy efficiency [J]. Wireless Personal Communications, 2015, 81(1): 373-386.

[5] S Chand, S Singh, B Kumar. Heterogeneous heed protocol for wireless sensor networks [J]. Wireless Personal Communications, 2014, 77(3): 2117-2139.

[6] R E. Blace, M Eltoweissy, W Abd-Almageed. Threat-Aware clustering in wireless sensor networks [D]. Proceedings of the 2008 IFIP Conference on Wireless Sensor and Actor Networks (WSAN 08), Ottawa, Ontario, Canmada, July 14-15, 2008.

[7] Bin Guo, Zhe Li. A dynamic-clustering reactive routing algorithm for wireless sensor networks [J].Wireless Networks, 2009, 15(4): 423430.

[8] S Kumar, S Kumar, S Nandi. Multi-Density Custering Algorithm for Anomaly Detection Using kdd'99 dataset [D]. First International Conference, ACC 2011, Kochi, India, July 22-24, 2011. Proceedings, Part I.

[9] P Ghosh, J Banerjee, S K Mirta etal. Sequential multi-clustering protocol using a node deployment protocol for efficient multiclustering in wireless sensor networks [D]. 4th International Conference, CNSA 2011, Chennai, India, July 15-17, 2011.

[10] SUN Yu-geng*, ZHOU Yin, BIAN Gui-nian etal. Energy-aware hierarchical clustering algorithm for wireless sensor networks [J]. Chinese Journal of Sensors and Actuators, 2007, 20(2):377-381.

[11] Chao-Tung Yang, Kuan-Chou Lai, Hao-Yu Tung. On construction of a well-balanced allocation strategy for heterogeneous multicluster computing environments [J].The Journalof Supercomputing2011, 56(3):270-299.

[12] H B Mitchell. Data Fusion: Concepts and Ideas [M]. Springer Berlin Heidelberg, 2012. 